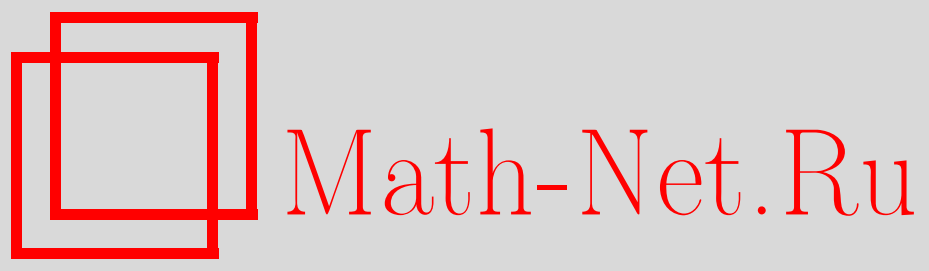

В. В. Козлов, Д. В. Трещёв, Тонкая и грубая энтропия в задачах статистической механики, ТMФ, 2007, том 151 , номер 1, 120-137

DOI: https://doi.org/10.4213/tmf6015

Использование Общероссийского математического портала Math-Net.Ru подразумевает, что вы прочитали и согласны с пользовательским соглашением http://www . mathnet.ru/rus/agreement

Параметры загрузки:

IP: 3.80 .181 .102

26 апреля 2023 г., 16:49:41

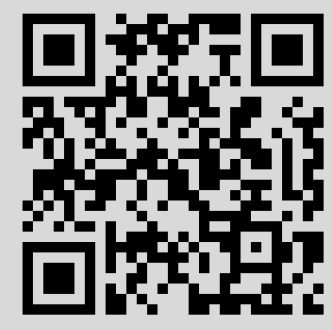




\section{ТОНКАЯ И ГРУБАЯ ЭНТРОПИЯ В ЗАДАЧАХ СТАТИСТИЧЕСКОЙ МЕХАНИКИ}

Рассматриваются динамические системы с фазовым пространством $\Gamma$, сохраняющие меру $\mu$. Разбиение $\Gamma$ на куски конечной $\mu$-меры порождает грубую энтропию - функционал на пространстве вероятностных мер на Г, обобщающий обычную (тонкую) энтропию Гиббса. Изучаются аппроксимационные свойства грубой энтропии при измельчении разбиения, а также свойства грубой энтропии как функции времени.

Ключевые слова: инвариантная мера, энтропия Гиббса, грубая энтропия.

\section{1. ОПРЕДЕЛЕНИЯ}

Пусть ( $Г$, dist) - метрическое пространство с мерой $\mu$, а $\nu$ - вероятностная мера с измеримой плотностью $\rho>0$ :

$$
d \nu=\rho d \mu, \quad \nu(\Gamma)=\int_{\Gamma} \rho d \mu=1 .
$$

В частности, $\rho \in L_{1}(\Gamma, \mu)$.

Пусть $\left\{\Gamma_{j}\right\}_{j \in J}-$ разбиение $\Gamma$ на измеримые подмножества

$$
\gamma_{j}=\mu\left(\Gamma_{j}\right), \quad 0<\mu\left(\Gamma_{j}\right)<\infty, \quad j \in J
$$

Множество $J$ считается конечным или счетным. Величину

$$
\sup _{j \in J}\left(\operatorname{diam} \Gamma_{j}\right) \leqslant \infty
$$

(диаметр берется в метрике пространства $Г$ ) назовем диаметром разбиения $\left\{\Gamma_{j}\right\}$. Положим

$$
\rho_{j}=\frac{\lambda_{j}}{\gamma_{j}}, \quad \lambda_{j}=\int_{\Gamma_{j}} \rho d \mu, \quad \sum_{j \in J} \lambda_{j}=1,
$$

* Математический институт им. В. А. Стеклова РАН, Москва, Россия. E-mail: kozlov@pran.ru

${ }^{\dagger}$ Московский государственный университет им. М. В. Ломоносова, Москва, Россия. E-mail: treschev@mi.ras.ru 
и рассмотрим новую плотность $\bar{\rho}: \Gamma \rightarrow \mathbb{R}$ такую, что $\left.\bar{\rho}\right|_{\Gamma_{j}}=\rho_{j}, \quad j \in J$. Будем называть $\bar{\rho}$ грубой плотностью. Соответствующая мера $\bar{\nu}, d \bar{\nu}=\bar{\rho} d \mu$, также является вероятностной, поскольку

$$
\int_{\Gamma} \bar{\rho} d \mu=\sum_{j \in J} \int_{\Gamma_{j}} \rho_{j} d \mu=\sum_{j \in J} \rho_{j} \gamma_{j}=1 .
$$

Определим функционал $\mathbf{S}$ такой, что для любой неотрицательной $\mu$-измеримой функции $\alpha: \Gamma \rightarrow \mathbb{R}$

$$
\mathbf{S}(\alpha)=-\int_{\Gamma} \alpha \ln \alpha d \mu
$$

при условии, что интеграл сходится к некоторой конечной или бесконечной величине. Как обычно, функцию $\alpha \ln \alpha$ доопределяем нулем при $\alpha=0$.

Определим тонкую энтропию $s=\mathbf{S}(\rho)$, а также грубую энтропию $\bar{s}=\mathbf{S}(\bar{\rho})$. Выполнено равенство

$$
\bar{s}=-\sum_{j \in J} \gamma_{j} \rho_{j} \ln \rho_{j}=-\sum_{j \in J} \lambda_{j} \ln \lambda_{j}+\sum_{j \in J} \lambda_{j} \ln \gamma_{j} .
$$

В частности, если все $\gamma_{j}$ равны друг другу, имеем

$$
\bar{s}=-\sum_{j \in J} \lambda_{j} \ln \lambda_{j}+\ln \gamma, \quad \gamma=\gamma_{j}, \quad j \in J,
$$

так что с точностью до аддитивной постоянной (зависящей только от $\gamma$ ) грубая энтропия совпадает с информационной энтропией $-\sum \lambda_{j} \ln \lambda_{j}$. Отметим, что формула для энтропии вида (1.1) в случае дискретного распределения вероятностей используется в теории равновесных состояний (см., например, монографию [1]).

При фиксированных $\gamma_{j}$ максимальное значение (1.1) достигается при

$$
\lambda_{j}=\frac{\gamma_{j}}{\sum_{i \in J} \gamma_{i}}
$$

при условии $\mu(\Gamma)<\infty$. Если мера $\mu$ фазового пространства бесконечна и все $\gamma_{j}$ ограничены, то $\sup \bar{s}=+\infty$.

Как установил Гиббс, справедливо неравенство

$$
s \leqslant \bar{s}
$$

Оно является простым следствием неравенства Йенсена для выпуклой функции $\rho \ln \rho$ (см. поучительное обсуждение в монографии [2]).

\section{2. ПРИМЕР ОТСУТСТВИЯ АППРОКСИМАЦИИ}

Если $\mu(\Gamma)=\infty$, то, вообще говоря, грубая энтропия не аппроксимирует тонкую, даже если диаметр разбиения сколь угодно мал. 
ПримеР 1. Пусть $\Gamma=\mathbb{R}$ с мерой Лебега. Пусть $\left\{a_{n}\right\}_{n \in \mathbb{N}}-$ последовательность такая, что

$$
0 \leqslant a_{n}<1, \quad \sum_{n=1}^{\infty} a_{n}=1, \quad \sum_{n=1}^{\infty} a_{n} \ln a_{n}=-\infty .
$$

В качестве $a_{n}$ можно взять, например, $c /\left(n \ln ^{1+\varepsilon} n\right), 0<\varepsilon<1$.

Рассмотрим вероятностную меру $\nu$ с плотностью

$$
\rho(x)= \begin{cases}1, & \text { если } x \in\left[n, n+a_{n}\right] \text { для некоторого } n \in \mathbb{N}, \\ 0 & \text { для остальных } x .\end{cases}
$$

Тонкая энтропия $s$ такой меры, очевидно, равна нулю.

Для любого целого $K>0$ рассмотрим разбиение

$$
\Gamma_{j}=\left\{x \in \mathbb{R}: \frac{j}{K} \leqslant x<\frac{(j+1)}{K}\right\}, \quad j \in \mathbb{Z} .
$$

Диаметр разбиения $\left\{\Gamma_{j}\right\}$ равен $1 / K$.

ПрЕДЛОЖЕНИЕ. Для любого целого $K>0$ грубая энтропия $\bar{s}$ равна $+\infty$.

ДокАЗАТЕЛЬСтво. Имеем равенство

$$
\bar{s}=-\sum_{j \in \mathbb{Z}} \frac{1}{K} \rho_{j} \ln \rho_{j}, \quad \rho_{j}=K \int_{j / K}^{(j+1) / K} \rho(x) d x \leqslant 1 .
$$

Пусть $N \in \mathbb{N}$ таково, что для всех $n>N$ выполнено неравенство $a_{n}<1 / K$. Тогда для $n>N$ получаем $\rho_{K n}=K a_{n}$.

Так как $\rho_{j} \ln \rho_{j}<0$ для всех $j \in \mathbb{Z}$, имеем оценку

$$
\bar{s} \geqslant-\sum_{n>N} \frac{1}{K} \rho_{K n} \ln \rho_{K n}=-\sum_{n>N} a_{n} \ln \left(K a_{n}\right)=+\infty,
$$

что и требовалось доказать.

Это утверждение опровергает расхожее представление о приближении грубой энтропии к тонкой при измельчении разбиения (ср. с [3], [4]). Стоит еще отметить, что в случае $\mu(\Gamma)=\infty$ грубая плотность, вообще говоря, не стремится к тонкой (в норме $\left.L_{1}=L_{1}(\Gamma, \mu)\right)$ при неограниченном уменьшении диаметра разбиения. Более того, грубая плотность не стремится к тонкой даже в слабом смысле. Слабая сходимость последовательности функций $\rho_{n}$ из $L_{1}$ к функции $\rho \in L_{1}$ означает, что

$$
\int_{\Gamma} \rho_{n} \varphi d \mu \rightarrow \int_{\Gamma} \rho \varphi d \mu
$$

для любой пробной функции $\varphi \in L_{\infty}(\Gamma, \mu)$. 


\section{3. АППРОКСИМАЦИОННЫЕ ТЕОРЕМЫ}

3.1. Компактный случай. В компактном случае грубая энтропия (плотность), как правило, приближает тонкую. Основным требованием здесь является согласованность на Г структур метрического и измеримого пространств. А именно, мы предполагаем, что пространство $C^{0}(\Gamma)$ непрерывных функций на Г плотно в $L_{1}(\Gamma, \mu)$.

Теорема 1. Предположим, что пространство $Г$ компактно, $\mu(\Gamma)=1, C^{0}(\Gamma)$ плотно в $L_{1}(\Gamma, \mu)$. Тогда при неограниченном уменъшении диаметра разбиения $\left\{\Gamma_{j}\right\}$ плотность $\bar{\rho}$ с любой наперед заданной точностъю аппроксимирует $\rho$ в метpuке $L_{1}(\Gamma, \mu)$.

Иначе говоря, при неограниченном уменьшении диаметра разбиения грубая плотность слабо сходится к тонкой плотности. Последнее свойство представляется существенным при переходе от микро- к макроописанию (к исследованию эволюции средних значений динамических величин).

Доказательство теоремы 1 приведено в приложении (см. п. П.1).

Теорема 2. Предположим, что пространство Г компактно, $\mu(\Gamma)=1, C^{0}(\Gamma)$ плотно в $L_{1}(\Gamma, \mu) u|s|<\infty$. Тогда при неограниченном уменвшении диаметра разбиения $\left\{\Gamma_{j}\right\}$ энтропия $\bar{s}$ с любой наперед заданной точностью аппроксимирует $s$.

Доказательство теоремы 2 опирается на две леммы.

ЛЕмма 1. Теорема 2 верна при дополнительном предположении $\rho<\Delta$ для некоторого $\Delta>1$.

Пусть, как обычно, $e$ - основание натурального логарифма.

ЛЕмма 2. Теорема 2 верна при дополнителъном предположении $\delta<\rho<\Delta д л я$ некоторых $\delta \in(0,1 / e) u \Delta>1$.

Для доказательства теоремы 2 докажем

импликацию лемма $1 \Longrightarrow$ теорема 2 (см. приложение, п. П.2),

импликацию лемма $2 \Longrightarrow$ лемма 1 (см. приложение, п. П.3) и

лемму 2 (см. приложение, п. П.4).

Отметим, что в теоремах 1 и 2 условие $\sup _{j \in J} \operatorname{diam}\left(\Gamma_{j}\right) \rightarrow 0$ нельзя заменить более слабым условием $\sup _{j \in J} \mu\left(\Gamma_{j}\right) \rightarrow 0$. Приведем простой пример. Пусть $\Gamma-$ квадрат

$$
\left\{(x, y) \in \mathbb{R}^{2}: 0 \leqslant x \leqslant 1,0 \leqslant y \leqslant 1\right\},
$$

$\mu$ - стандартная мера Лебега на $\Gamma, \rho(x, y)=y$, а измеримые куски $\Gamma_{j}$ разбиения $\Gamma-$ полоски

$$
\left\{\frac{j-1}{N} \leqslant x \leqslant \frac{j}{N}, \quad 0 \leqslant y \leqslant 1\right\}, \quad j=1, \ldots, N .
$$

Тогда $\lim _{N \rightarrow \infty} \mu\left(\Gamma_{j}\right)=0$, однако диаметр $\Gamma_{j}$ к нулю не стремится. Легко показать, что для типичной (в любом разумном смысле) плотности $\rho(x, y)$

$$
\lim _{N \rightarrow \infty} \bar{\rho} \neq \rho, \quad \lim _{N \rightarrow \infty} \bar{s} \neq s .
$$


3.2. Некомпактный случай. Если плотность $\rho$ достаточно быстро стремится к нулю на бесконечности, грубая энтропия аппроксимирует тонкую и в некомпактном случае. Чтобы сформулировать точный результат, нам потребуются некоторые определения.

ОПРЕДЕЛЕНИЕ 1 . Пространство $(\Gamma, \mu$, dist) имеет тип $n$, если существует последовательность компактов $K_{0}, K_{1}, \ldots$ таких, что

а) $K_{0} \subset K_{1} \subset \cdots \subset \Gamma$;

б) $\Gamma=\bigcup_{l=0}^{\infty} K_{l}$;

в) $\mu\left(K_{0}\right)<\infty, \mu\left(K_{l+1} \backslash K_{l}\right) \leqslant C l^{n-1}$ для некоторой постоянной $C>0$;

г) для любых точек $x \in K_{l}, y \in K_{s}$ неравенство $\operatorname{dist}(x, y)<1$ возможно лишь в случае $|l-s| \leqslant 1$.

Простейшим примером пространства типа $n$ является $\mathbb{R}^{n}$ с мерой Лебега и евклидовой метрикой. В качестве компактов $K_{j}$ здесь можно взять шары радиусов $j$ с общим центром.

Теорема 3. Предположим, что

1) пространство ( $\Gamma, \mu$, dist) uмеет mun $n$;

2) $C^{0}\left(K_{l}\right)$ плотно в $L_{1}\left(K_{l}, \mu\right)$ для всех $l=0,1, \ldots$;

3) $\left.\rho\right|_{K_{l}}<c_{\rho} l^{-n-\delta}, l=0,1, \ldots$, где $c_{\rho} u \delta$ - положительные постоянные;

4) $|s|<\infty$.

Тогда при неограниченном уменьшении диаметра разбиения $\left\{\Gamma_{j}\right\}$ энтропия $\bar{s}$ c любой наперед заданной точностью аппроксимирует $s$.

Доказательство теоремы 3 приведено в приложении (п. П.5).

\section{4. ПРОБЛЕМА СТАБИЛИЗАЦИИ ПЛОТНОСТИ}

Пусть теперь $\Gamma$ - фазовое пространство динамической системы, задаваемой потоком (однопараметрической группой преобразований) $g^{t}, t \in \mathbb{R}$, сохраняющим меру $\mu$. Рассмотрим вероятностную меру $\nu=\nu^{t}$ с $\mu$-измеримой плотностью $\rho=\rho^{t} \geqslant 0$; $d \nu^{t}=\rho^{t} d \mu$. По определению для любого $t \in \mathbb{R}$ и любого измеримого множества $D \subset \Gamma$

$$
\nu^{t}(D)=\nu^{0}\left(g^{-t}(D)\right) .
$$

Тогда $\rho^{t}=\rho^{0} \circ g^{t}$. Если $\Gamma$ - гладкое многообразие, а $g^{t}$ - поток, задаваемый векторным полем $v$, то в локальных координатах $\rho^{t}$ удовлетворяет уравнению Лиувилля ${ }^{1)}$

$$
\frac{\partial \rho^{t}}{\partial t}-\operatorname{div}\left(\rho^{t} v\right)=0
$$

Грубая плотность $\bar{\rho}^{t}$ определяется следующим образом:

$$
\left.\bar{\rho}^{t}\right|_{\Gamma_{j}}=\rho_{j}(t)=\frac{1}{\gamma_{j}} \int_{\Gamma_{j}} \rho^{t} d \mu .
$$

\footnotetext{
1) Традиционно в уравнении Лиувилля перед вторым слагаемым стоит знак плюс. Уравнение, используемое здесь, получается из стандартного уравнения Лиувилля в результате замены $t \mapsto-t$.
} 
В первую очередь нас будут интересовать гамильтоновы системы, хотя многие результаты справедливы и для систем более общего вида. В гамильтоновом случае $\mu$ - инвариантная мера Лиувилля - элемент объема фазового пространства. Для натуральных систем, конечно, $\mu(\Gamma)=\infty$, но ограничение $\mu$ на уровень энергии может оказаться конечной мерой.

В конструкциях, связанных с энтропией Гиббса, важную роль играет существование пределов

$$
\lim _{t \rightarrow+\infty} \rho^{t}, \quad \lim _{t \rightarrow-\infty} \rho^{t} .
$$

Указанные пределы следует понимать в смысле слабой сходимости в одном из функциональных пространств, важнейшими из которых в данном контексте являются пространства $L_{1}(\Gamma, \mu)$ и $L_{2}(\Gamma, \mu)$. Существование аналогичных пределов для грубых плотностей $\bar{\rho}^{t}$ также представляет значительный интерес.

Хорошо известно, что плотность $\rho^{t}$, вообще говоря, не имеет предела при $t \rightarrow \infty$. Это обстоятельство является основным препятствием к обоснованию "нулевого" начала термодинамики в теории ансамблей Гиббса. Одна из попыток преодоления этой трудности состоит во введении грубой плотности $\bar{\rho}^{t}$, порожденной разбиением $\left\{\Gamma_{j}\right\}$ фазового пространства. Гиббс пытался доказать (см. монографию [5], гл. ХІІ), что в типичном случае грубая плотность $\bar{\rho}^{t}$ сходится при $t \rightarrow \infty$ к некоторой функции, зависящей лишь от полной энергии гамильтоновой системы. "Пытаться доказать это утверждение почти безнадежно; оно является более сильным, чем эргодическая теорема. Известные доводы самого Гиббса (основанные на аналогиях с перемешиванием жидкостей), даже если отбросить содержащиеся в них существенные ошибки, служат в лучшем случае указанием на правдоподобность этой “теоремы" " (см. монографию [2], гл. III).

Впрочем, как замечает сам Гиббс, для линейных гамильтоновых систем грубая плотность $\bar{\rho}^{t}$ осциллирует и вообще не имеет предела при неограниченном возрастании времени. С другой стороны, предположение Гиббса заведомо справедливо для гамильтоновых систем с перемешиванием на изоэнергетических поверхностях. Это наблюдение принадлежит Крылову [3], однако он не заметил важного обстоятельства: для систем с перемешиванием пределы грубой плотности $\bar{\rho}^{t}$ при $t \rightarrow-\infty$ и $t \rightarrow+\infty$ coвnадают. Такая симметрия прошлого и будущего (вытекающая из обратимости натуральных гамильтоновых систем) противоречит традиционным представлениям об однонаправленности приближения изолированной системы к состоянию теплового равновесия.

Ниже мы обсуждаем предположение Гиббса о приближении к тепловому равновесию (в несколько ослабленной формулировке) квазиоднородных гамильтоновых систем. Напомним, что гамильтонова система

$$
\dot{x}_{j}=\frac{\partial H}{\partial y_{j}}, \quad \dot{y}_{j}=-\frac{\partial H}{\partial x_{j}}, \quad j=1, \ldots, n,
$$

называется квазиоднородной, если существуют вещественные постоянные (веса́ квазиоднородности) $\alpha, \beta, \gamma, \alpha+\beta+1=\gamma$, такие, что для всех $\lambda>0$

$$
H\left(\lambda^{\alpha} x, \lambda^{\beta} y\right)=\lambda^{\gamma} H(x, y) .
$$


Иначе говоря, уравнения Гамильтона инвариантны при подстановках

$$
t \mapsto \frac{t}{\lambda}, \quad x \mapsto \lambda^{\alpha} x, \quad y \mapsto \lambda^{\beta} y
$$

Как обычно, в качестве инвариантной меры $\mu$ берется мера Лиувилля, имеющая единичную плотность в координатах $(x, y)$.

Приведем два примера.

ПримеР 2. Системы с однородным потенциалом

$$
H=\frac{1}{2} \sum y_{j}^{2}+V_{m}(x)
$$

где $m$ - степень однородности потенциальной энергии $V_{m}$. Здесь

$$
\alpha=\frac{2}{m-2}, \quad \beta=\frac{m}{m-2}, \quad \gamma=\frac{2 m}{m-2} .
$$

В частности, сюда относится задача $n$ тел с ньютоновым потенциалом $(\alpha=-2 / 3$, $\beta=1 / 3, \quad \gamma=2 / 3)$.

Исключительный случай $m=2$ соответствует линейным системам, которые не являются квазиоднородными.

ПримеР 3. Движение по инерции:

$$
H=\frac{1}{2} \sum a_{j k}(x) y_{j} y_{k}, \quad x \in M,
$$

где $M$ - гладкое риманово многообразие. Здесь $\alpha=0, \beta=1, \gamma=2$. Сюда же относятся бильярдные системы, в которых $M$ - многообразие с краем, и отражение от края упругое.

Теорема 4. Пусть гамильтонова система (4.2) квазиоднородна, начальная

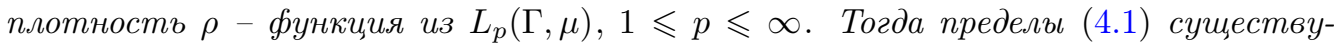
ют (в смысле слабой $L_{p}(\Gamma, \mu)$-сходимости) и совпадают.

Сделаем несколько замечаний.

1. Теорема 4 получена в работе [6]. Она справедлива для более общего класса динамических систем (не обязательно гамильтоновых) - cлоистых потоков, введенных в работе [7].

2. Пусть $\mathcal{A}^{k}$ - класс систем гладкости $k$, в которых пределы (4.1) существуют для любой плотности $\rho \in L_{p}(\Gamma, \mu)$.

ГипотезА. Для любого достаточно большого $k$ (включая $k=\infty u k=\omega$ ) множество $\mathcal{A}^{k}$ состоит из систем общего положения в пространстве систем гладкости $k$.

По поводу справедливости этой гипотезы известно довольно мало. Если на $(\Gamma, \mu)$ имеется перемешивающая гиперболическая система (система Аносова), то внутренность $\mathcal{A}^{k}$ в $C^{k}$-топологии не пуста. В самом деле, гиперболические перемешивающие системы, очевидно, лежат в $\mathcal{A}^{k}$ и, как известно, являются структурно-устойчивыми. 
$\mathrm{K}$ сожалению, не следует ожидать, что условие общности положения в гипотезе можно понимать в том смысле, что $\mathcal{A}^{k}$ открыто и всюду плотно при $k<\omega$. Причина состоит в том, что согласно работе [8] гладкую систему с гомоклиническим касанием можно как угодно точно в $C^{\infty}$-топологии приблизить системой, имеющей инвариантное множество, на котором динамика является жестким поворотом. Для такой системы пределы (4.1) не существуют для большинства начальных плотностей $\rho$. (Строго говоря, результаты работы [8] получены для отображений, но не вызывает сомнений возможность применения аналогичных методов и в случае потоков.)

Таким образом, общность положения в гипотезе, по-видимому, следует понимать в том смысле, что $\mathcal{A}^{k}$ является подмножеством второй категории Бэра.

3. Если гамильтонова система эргодична на изоэнергетических многообразиях, то состояние статистического равновесия, задаваемое стационарной инвариантной мерой $d \nu^{\infty}=\rho^{\infty} d \mu$, будет микроканоническим (плотность $\rho^{\infty}$ зависит лишь от полной энергии). Этому свойству мера $\bar{\nu}^{\infty}$, вообе говоря, не удовлетворяет: она даже не инвариантна относительно фазового потока.

4. В случае дискретных динамических систем конструкции аналогичны. В самом деле, пусть $g: \Gamma \rightarrow \Gamma$ - автоморфизм (или эндоморфизм) измеримого пространства $(\Gamma, \mu)$, т.е. для любого $\mu$-измеримого множества $D \subset \Gamma$

$$
\mu(D)=\mu\left(g^{-1}(D)\right)
$$

Как обычно, $g^{-1}(D)$ - полный прообраз $D$ при отображении $g$.

Если $\rho^{0}: \Gamma \rightarrow \mathbb{R}$ - плотность вероятностной меры $\nu^{0}, d \nu^{0}=\rho^{0} d \mu$, то при целых $n \geqslant 0$ имеем меру $\nu^{n}$ :

$$
d \nu^{n}=\rho^{n} d \mu, \quad \rho^{n}=\rho^{0} \circ g^{n} .
$$

Тогда для любого $\mu$-измеримого множества $D \subset \Gamma$

$$
\nu^{n}(D)=\nu^{0}\left(g^{-n}(D)\right)
$$

\section{5. СТАБИЛИЗАЦИЯ ГРУБОЙ ПЛОТНОСТИ}

Гиббс пытался доказать, что грубая энтропия $\bar{s}_{t}$ возрастает с возрастанием времени. Однако его рассуждения также оказались некорректными (их анализ содержится в монографии [3], где имеются другие ссылки). Интересно отметить, что поначалу этот неверный результат многими авторами воспринимался всерьез. Например, Пуанкаре [4] пишет о нем как о хорошо известном факте. Грубую энтропию следует отличать от энтропии Больцмана, связанной со статистикой в $\mu$-пространстве.

Некоторую информацию о поведении грубой плотности $\bar{\rho}^{t}$ при $t \rightarrow \infty$ дает следующая теорема.

Теорема 5. Пусть гамилътонова система (4.2) квазиоднородна, начальная плотность $\rho$ - функция из $L_{p}(\Gamma, \mu), 1 \leqslant p \leqslant \infty, u\left\{\Gamma_{j}\right\}$ - разбиение фазового пространства на куски конечной меры Лиувилля. Тогда $\lim _{t \rightarrow+\infty} \rho_{j}(t) u \lim _{t \rightarrow-\infty} \rho_{j}(t)$ существуют в смысле слабой $L_{p}$-сходимости и совпадают. 
Теорема 5 легко выводится из теоремы 4. Действительно, пусть $g^{t}-$ фазовый поток системы (4.2). Тогда $\rho^{t}=\rho \circ g^{t} \in L_{p}(\Gamma, \mu)$ при всех $t \in \mathbb{R}$. Пусть $\varphi_{j}-$ характеристическая функция измеримой области $\Gamma_{j}$. Так как $\rho^{t}$ слабо сходится к $\rho^{\infty}$ при $t \rightarrow \pm \infty$ и $\rho^{\infty} \in L_{p}(\Gamma, \mu)$, то

$$
\rho_{j}(t)=\frac{1}{\gamma_{j}} \int_{\Gamma} \rho^{t} \varphi_{j} d \mu \rightarrow \frac{1}{\gamma_{j}} \int_{\Gamma} \rho^{\infty} \varphi_{j} d \mu .
$$

Остается заметить, что $\int_{\Gamma} \bar{\rho}^{\infty} d \mu=\int_{\Gamma} \rho^{\infty} d \mu$ и $\bar{\rho}^{\infty} \in L_{p}(\Gamma)$.

Как показано в работе [7], если уровни энергии квазиоднородной гамильтоновой системы компактны, то $\rho^{\infty}$ - плотность некоторой вероятностной меры:

$$
\int_{\Gamma} \rho^{\infty} d \mu=1
$$

Следовательно, в этом случае функция $\bar{\rho}^{\infty}$ из теоремы 5 также задает вероятностную меру.

Рассмотрим квазиоднородную гамильтонову систему (4.2) на инвариантном куске

$$
\Gamma=\left\{(x, y): h_{1} \leqslant H(x, y) \leqslant h_{2}\right\}, \quad h_{1}<h_{2} .
$$

Пусть Г компактно. Так как $C^{0}(\Gamma)$ плотно в $L_{1}(\Gamma, \mu)\left(\mu-\right.$ мера Лиувилля $\left.d^{n} x d^{n} y\right)$, то из теорем 1 и 5 вытекает

СледствиЕ. Пусть начальная плотность $\rho: \Gamma \rightarrow \mathbb{R}-$ функция из $L_{1}(\Gamma, \mu)$ и $\left\{\Gamma_{j}\right\}$ - разбиение множества (5.2). Тогда при $\sup \left(\operatorname{diam} \Gamma_{j}\right) \rightarrow 0$ плотность $\bar{\rho}^{\infty}$ с любой наперед заданной точностью аппроксимирует слабый предел $\rho^{\infty}$ в метрике $L_{1}(\Gamma, \mu)$.

Действительно, правая часть предельного соотношения (5.1) совпадает с $\left(\bar{\rho}^{\infty}\right)_{j}$. Таким образом, плотность $\bar{\rho}^{\infty}$ получается из плотности $\rho^{\infty}$ усреднением по ячейкам разбиения $\Gamma=\cup \Gamma_{j}$.

\section{6. ТЕОРЕМА О ВОЗРАСТАНИИ ГРУБОЙ ЭНТРОПИИ}

Пусть $\rho^{\infty}$ - слабый предел плотности $\rho^{t}$ при $t \rightarrow \pm \infty\left(\rho_{0}=\rho\right)$. Тогда (как установлено в работе [7]) имеет место неравенство

$$
S\left(\rho^{\infty}\right) \geqslant S(\rho)
$$

Оказывается, что, вопреки распространенному мнению (высказанному впервые Гиббсом и поддержанному Пуанкаре), грубая энтропия не всегда возрастает. Это показывает простой пример.

ПримеР 4. Рассмотрим вертикально расположенный отрезок длины $l$ в поле сил тяжести и ансамбль частиц, которые упруго отражаются от его концов. Если квадрат скорости частицы превосходит $2 g l$ ( $g$ - ускорение свободного падения), то частица периодически сталкивается с обоими концами отрезка. Пусть начальная плотность $\rho$ постоянна в прямом произведении $Г$ отрезка $0 \leqslant x \leqslant l$ и области 
$V=\left\{v: 2 g l \leqslant v^{2} \leqslant c, c=\right.$ const $\}$ на оси скоростей. Рассмотрим разбиение $Г$ на два одинаковых куска

$$
\Gamma_{1}=\left\{0 \leqslant x \leqslant \frac{l}{2}\right\}, \quad \Gamma_{1}=\left\{\frac{l}{2} \leqslant x \leqslant l\right\} .
$$

Начальная энтропия вычисляется по формуле (1.1), в которой $\lambda_{1}=\lambda_{2}=1 / 2$ и $\gamma_{j}=\mu\left(\Gamma_{1}\right)=\mu\left(\Gamma_{2}\right)$. Легко понять, что в стационарном состоянии (когда плотность $\rho^{t}$ заменяется слабым пределом) бо́льшая часть частиц из ансамбля будет расположена в верхней половине отрезка (поскольку в этой половине частицы движутся с меньшей скоростью). Следовательно, при $\rho=\rho^{\infty}$ в формуле (1.1) уже $\lambda_{1} \neq \lambda_{2}$. Но тогда, как известно, $S\left(\rho^{\infty}\right)<S(\rho)$. Легко понять, что тот же вывод справедлив и в более общем случае, когда разбиение Г порождается разбиением отрезка на $n \geqslant 2$ равных частей.

Чтобы указать достаточные условия возрастания грубой энтропии, снова рассмотрим квазиоднородную систему уравнений Гамильтона (4.2), ограниченную на компактную инвариантную область (5.2).

Теорема 6. Пусть начальная плотность $\rho: \Gamma \rightarrow \mathbb{R}-$ функиия из $L_{1}(\Gamma, \mu)$ u $\left\{\Gamma_{j}\right\}$ - разбиение Г. Если неравенство (6.1) строгое, $S\left(\bar{\rho}^{\infty}\right)<\infty$, то при достаточно малых $\sup \left(\operatorname{diam} \Gamma_{j}\right)$ справедливо неравенство $\mathbf{S}\left(\bar{\rho}^{\infty}\right)>\mathbf{S}(\bar{\rho})$.

Это утверждение сразу доказывается с помощью следствия, приведенного в предыдущем разделе, и аппроксимационной теоремы 2: так как в нашем случае $C^{0}(\Gamma)$ плотно в $L_{1}(\Gamma, \mu)$ и $\mathbf{S}(\rho)<\mathbf{S}\left(\rho^{\infty}\right)<\infty$, то при $\sup \left(\operatorname{diam} \Gamma_{j}\right) \rightarrow 0$ разности $\mathbf{S}(\bar{\rho})$ и $\mathbf{S}(\rho)$, а также $\mathbf{S}\left(\bar{\rho}^{\infty}\right)$ и $\mathbf{S}\left(\rho^{\infty}\right)$ сколь угодно малы.

\section{7. ИНТЕГРИРУЕМЫЕ СИСТЕМЫ}

Рассмотрим интегрируемую по Лиувиллю гамильтонову систему с компактными совместными уровнями первых интегралов. В области, не содержащей критических интегральных уровней, такая система может быть записана в переменных “действие-угол":

$$
\dot{x}=\omega(y), \quad \dot{y}=0, \quad \mathbb{T}^{n}=\left\{x=\left(x_{1}, \ldots, x_{n}\right) \bmod 1\right\}, \quad y \in D \subset \mathbb{R}^{n} .
$$

Если зависимость частот $\omega$ от действий $y$ невырожденна $($ т.е. $\operatorname{det}(\partial \omega / \partial y) \neq 0)$, то по крайней мере локально $\omega$ можно взять вместо $y$ в качестве фазовых координат. Тогда система принимает вид

$$
\dot{x}=\omega, \quad \dot{\omega}=0 .
$$

Отметим, что (7.1) - квазиоднородные уравнения Гамильтона: $x_{j}, \omega_{j}$ - сопряженные канонические переменные, а $H=\left(\omega_{1}^{2}+\cdots+\omega_{n}^{2}\right) / 2$ - функция Гамильтона.

$\mathrm{K}$ уравнениям (7.1) можно прийти также из других соображений. Рассмотрим бесстолкновительную сплошную среду, заключенную в $n$-мерный сосуд - прямоугольный параллелепипед. Предполагается, что частицы упруго отражаются от

5 Теоретическая и математическая физика, т. 151, № 1, 2007 г. 
стенок сосуда - границы параллелепипеда - и не сталкиваются друг с другом, поэтому такую среду можно назвать идеалъным газом. Такую модель одномерного идеального газа впервые рассмотрел Пуанкаре [4]. Все это, конечно, является частным случаем общей теории ансамблей Гиббса. Как заметил Пуанкаре, после перехода к $2^{n}$-листному накрытию параллелепипеда тором $\mathbb{T}^{n}$ уравнение движения частиц совпадет с системой (7.1).

Уравнения (7.1) имеют инвариантную меру $d \mu=d x d \omega$ в фазовом пространстве $\Gamma=\mathbb{T}^{n} \times \mathbb{R}^{n}$. Пусть $\rho(x, \omega)$ - плотность вероятностной меры $\nu: d \nu=\rho d \mu$. Для любой пары натуральных чисел $N, M$ рассмотрим разбиение фазового пространства $\Gamma$ на части $\Gamma_{j k}, j \in \mathbb{Z}^{n} / N \mathbb{Z}^{n}, k \in \mathbb{Z}^{n}$ :

$$
\begin{aligned}
\Gamma_{j k} & =\Gamma_{j}^{x} \times \Gamma_{k}^{\omega}, \\
\Gamma_{j}^{x} & =\left\{x \in \mathbb{T}^{n}: \frac{j_{l}}{N} \leqslant x_{l} \leqslant \frac{j_{l}+1}{N}, \quad l=1, \ldots, n\right\}, \\
\Gamma_{k}^{\omega} & =\left\{\omega \in \mathbb{R}^{n}: \frac{k_{l}}{M} \leqslant \omega_{l} \leqslant \frac{k_{l}+1}{M}, \quad l=1, \ldots, n\right\} .
\end{aligned}
$$

Мера $\mu$ каждого из кусков $\Gamma_{j k}$ равна $\mu\left(\Gamma_{j k}\right)=(N M)^{-n}$.

Подсчитаем значение грубой плотности на $\Gamma_{j k}$ :

$$
\rho_{j k}(t)=(N M)^{n} \int_{\Gamma_{j k}} \rho(x+\omega t, \omega) d x d \omega .
$$

Положим

$$
\langle\rho\rangle(\omega)=\int_{\mathbb{T}^{n}} \rho(x, \omega) d x, \quad\langle\rho\rangle_{k}=\int_{\Gamma_{k}^{\omega}}\langle\rho\rangle(\omega) d \omega .
$$

Очевидно, что $\langle\rho\rangle$ - плотность некоторой вероятностной меры на $Г$, причем для любого $t$ имеем $\langle\rho\rangle_{j k}(t)=\langle\rho\rangle_{k}$.

ТЕОРема 7. Предположим, что функция $\rho$ ограничена на Г и липшецева по переменным $\omega$. Тогда при $t>0$

$$
\begin{gathered}
\left|\rho_{j k}(t)-\langle\rho\rangle_{k}\right| \leqslant \frac{n M N^{n}}{t}\left(\frac{\|\rho\|_{1}}{M}+2\|\rho\|_{0}\right), \\
\|\rho\|_{0}=\sup _{\Gamma}|\rho|, \quad\|\rho\|_{1}=\sup \frac{\left|\rho\left(x, \omega^{\prime}\right)-\rho\left(x, \omega^{\prime \prime}\right)\right|}{\left|\omega^{\prime}-\omega^{\prime \prime}\right|},
\end{gathered}
$$

где $\left(x, \omega^{\prime}\right),\left(x, \omega^{\prime \prime}\right) \in \Gamma, \omega^{\prime} \neq \omega^{\prime \prime}$.

Доказательство теоремы 7 содержится в приложении (п. П.6).

Пусть функция $\rho$ финитна, $\bar{\rho}$ и $\overline{\langle\rho\rangle}$ - грубые плотности, соответствующие разбиению $\Gamma_{j k}$ и плотностям $\rho$ и $\langle\rho\rangle$, соответственно. Тогда

$$
\mathbf{S}(\bar{\rho})-\mathbf{S}(\overline{\langle\rho\rangle})=-\frac{1}{(M N)^{n}} \sum_{j, k}\left(\rho_{j k} \ln \rho_{j k}-\langle\rho\rangle_{k} \ln \langle\rho\rangle_{k}\right),
$$

причем лишь конечное число слагаемых в сумме отлично от нуля. По-видимому, в типичной ситуации следует ожидать, что при $t \rightarrow \infty$ разность $\mathbf{S}(\bar{\rho})-\mathbf{S}(\overline{\langle\rho\rangle})$ имеет порядок $1 / t$, хотя легко построить примеры, когда $\mathbf{S}(\bar{\rho})-\mathbf{S}(\overline{\langle\rho\rangle}) \sim t^{-1} \ln t$. 


\section{8. ПЕРЕМЕШИВАЮЩИЕ СИСТЕМЫ}

Динамику в системах, рассмотренных в разделе 7, принято называть регулярной. Ее антиподом является хаотическая динамика. Здесь прежде всего мы имеем в виду перемешивающие системы. Напомним

ОПРЕДЕЛЕНИЕ 2. Пусть поток $g^{t}$ на фазовом пространстве $Г$ сохраняет вероятностную меру $\mu$. Поток $g^{t}$ называется перемешивающим, если для любой пары функций $\varphi, \psi: \Gamma \rightarrow \mathbb{R}$ из достаточно широкого функционального пространства

$$
\lim _{t \rightarrow \infty} \int_{\Gamma} \varphi \circ g^{t} \psi d \mu=\int_{\Gamma} \varphi d \mu \int_{\Gamma} \psi d \mu .
$$

В случае гиперболических систем (систем Аносова) имеет место экспоненциальное убывание корреляций, т.е. для некоторых постоянных $C>0$ и $\tau \in(0,1)$

$$
\left|\lim _{t \rightarrow \infty} \int_{\Gamma} \varphi \circ g^{t} \psi d \mu-\int_{\Gamma} \varphi d \mu \int_{\Gamma} \psi d \mu\right|<C \tau^{|t|} .
$$

В этом случае грубая плотность всегда стремится к 1 при $t \rightarrow \infty$. Действительно,

$$
\rho_{j}(t)=\frac{1}{\gamma_{j}} \int_{\Gamma_{j}} \varphi \circ g^{t} d \mu=\frac{1}{\gamma_{j}} \int_{\Gamma} \varphi \circ g^{t} \chi_{\Gamma_{j}} d \mu,
$$

где $\chi_{\Gamma_{j}}$ - характеристическая функция множества $\Gamma_{j}$. Из (8.1) следует, что

$$
\rho_{j} \rightarrow \frac{1}{\gamma_{j}} \int_{\Gamma} \varphi d \mu \int_{\Gamma} \chi_{\Gamma_{j}} d \mu=1 .
$$

Более того, если выполнено неравенство (8.2), то $\rho_{j}$ приближается к 1 с экспоненциальной скоростью. Такого же поведения следует ожидать и от грубой энтропии: $\mathbf{S}\left(\bar{\rho}^{t}\right)$ экспоненциально быстро стремится к $\mathbf{S}(1)=0$.

Отметим, тем не менее, что к этим утверждениям следует относиться с известной долей осторожности, так как обычно функциональные пространства, для которых удается доказать соотношения (8.1), (8.2), обычно несколько у́же, чем пространство непрерывных функций на $\Gamma$, так что характеристические функции множеств $\Gamma_{j}$ в них не входят. По-видимому, основной причиной этого обстоятельства является не то, что функции $\chi_{\Gamma_{j}}$ слишком “плохие", а то, что их трудно включить в удобное (с точки зрения проверки (8.1), (8.2)) функциональное пространство. Впрочем, в конкретных примерах (скажем, для линейных гиперболических автоморфизмов тора) проверку приведенных в этом разделе утверждений о поведении грубой плотности и грубой энтропии легко провести непосредственно.

\section{ПРИЛОЖЕНИЕ}

П.1. Доказательство теоремы 1. Пусть $\varepsilon>0$ произвольно. Так как $C^{0}(\Gamma)$ плотно в $L_{1}(\Gamma, \mu)$, существует функция $\rho \in C^{0}(\Gamma)$ такая, что

$$
\left\|\rho-\rho_{c}\right\|<\varepsilon,
$$


где $\|\cdot\|-L_{1}$-норма. Имеем также простое неравенство

$$
\left\|\bar{\rho}-\bar{\rho}_{c}\right\| \leqslant\left\|\rho-\rho_{c}\right\|<\varepsilon .
$$

Функция $\rho_{c}$ равномерно непрерывна на $Г$. Поэтому существует $\delta>0$ такое, что

$$
\left|\rho_{c}\left(z_{1}\right)-\rho_{c}\left(z_{2}\right)\right|<\varepsilon
$$

для любых $z_{1}, z_{2} \in \Gamma, \operatorname{dist}\left(z_{1}, z_{2}\right)<\delta$. Следовательно $\left|\bar{\rho}_{c}-\rho_{c}\right|<\varepsilon$, откуда вытекает, что

$$
\left\|\bar{\rho}_{c}-\rho_{c}\right\|<\varepsilon .
$$

Комбинация (П.1) и (П.2) дает $\|\rho-\bar{\rho}\|<3 \varepsilon$.

П.2. Доказательство теоремы 2: часть 1. В этом пункте мы выводим теорему 2 из леммы 1 . Положим

$$
\begin{gathered}
\rho_{0}(x)=\left\{\begin{array}{lll}
\rho(x), & \text { если } & \rho(x) \leqslant \Delta, \\
\Delta, & \text { если } & \rho(x)>\Delta,
\end{array}\right. \\
\rho_{0 j}:=\left.\bar{\rho}_{0}\right|_{\Gamma_{j}}=\frac{1}{\gamma_{j}} \int_{\Gamma_{j}} \rho_{0} d \mu, \quad s_{0}=\mathbf{S}\left(\rho_{0}\right), \quad \bar{s}_{0}=\mathbf{S}\left(\bar{\rho}_{0}\right) .
\end{gathered}
$$

Из (П.2) следует, что $s_{0} \leqslant \bar{s}_{0}$.

Зафиксируем произвольное $\varepsilon>0$. Если $\Delta$ достаточно велико, то

$$
0 \leqslant s-s_{0} \leqslant \varepsilon
$$

Если диаметр разбиения $\left\{\Gamma_{j}\right\}$ достаточно мал, то согласно лемме 1 выполнены неравенства

$$
0<\bar{s}_{0}-s_{0} \leqslant \varepsilon
$$

Таким образом, достаточно доказать, что

$$
\bar{s}<\bar{s}_{0}+c \varepsilon|\ln \varepsilon|
$$

где $c>0$ - постоянная, не зависящая от $\varepsilon$. Действительно, тогда согласно (П.3) и (П.4) будем иметь $\left|s-\bar{s}_{0}\right|<2 \varepsilon$. Следовательно, из (П.2) и (П.5) вытекает, что

$$
s \leqslant \bar{s} \leqslant \bar{s}_{0}+c \varepsilon \ln \varepsilon \leqslant s+c \varepsilon \ln \varepsilon+2 \varepsilon,
$$

откуда получаем $|s-\bar{s}|<c \varepsilon \ln \varepsilon+2 \varepsilon$.

Оставшаяся часть пункта - доказательство оценки (П.5). Запишем (П.3) подробнеe:

$$
0<\int_{\Gamma \cap\{\rho \geqslant \Delta\}}\left(\rho \ln \rho-\rho_{0} \ln \rho_{0}\right) d \mu<\varepsilon .
$$

Следовательно,

$$
0<\int_{\Gamma \cap\{\rho \geqslant \Delta\}}\left(\rho \ln \Delta-\rho_{0} \ln \Delta\right) d \mu<\varepsilon
$$



откуда получаем

$$
0<\int_{\Gamma}\left(\rho-\rho_{0}\right) d \mu<\frac{\varepsilon}{\ln \Delta}
$$

Имеем неравенства

$$
0 \leqslant \rho_{0 j} \leqslant \rho_{j}, \quad \sum_{j}\left(\rho_{j}-\rho_{0 j}\right) \gamma_{j}<\frac{\varepsilon}{\ln \Delta} .
$$

Докажем вспомогательный факт: для любых $0 \leqslant a \leqslant b$ и $\sigma \in(0,1 / e)$ выполнено неравенство

$$
a \ln a \leqslant b \ln b+|\sigma \ln \sigma|+|1+\ln \sigma|(b-a) .
$$

В самом деле, если $a>\sigma$, то, пользуясь очевидным неравенством $\min _{\rho \geqslant \sigma}(\rho \ln \rho)^{\prime}=$ $1+\ln \sigma$, получаем

$$
a \ln a-b \ln b \leqslant|1+\ln \sigma|(b-a) .
$$

Если же $a \in(0, \sigma)$, имеем

$$
a \ln a-b \ln b \leqslant|a \ln a-\sigma \ln \sigma|+|\sigma \ln \sigma-b \ln b| \leqslant|\sigma \ln \sigma|+|1+\ln \sigma|(b-a) .
$$

Из неравенства (П.8) немедленно следует

$$
\sum_{j} \gamma_{j} \rho_{0 j} \ln \rho_{0 j} \leqslant \sum_{j} \gamma_{j} \rho_{j} \ln \rho_{j}+|\sigma \ln \sigma| \sum_{j} \gamma_{j}+\sum_{j}(|1+\ln \sigma|)\left(\rho_{j}-\rho_{0 j}\right) \gamma_{j}
$$

что с учетом (П.7) можно переписать в виде

$$
\bar{s} \leqslant \bar{s}_{0}+|\sigma \ln \sigma|+\frac{|1+\ln \sigma|}{\ln \Delta} \varepsilon .
$$

Теперь достаточно положить $\sigma=\varepsilon$.

П.3. Доказательство теоремы 2: часть 2. В этом пункте мы выводим лемму 1 из леммы 2. Пусть $\rho<\Delta$. Положим

$$
\begin{gathered}
\rho_{*}(x)=\left\{\begin{array}{lll}
\rho(x), & \text { если } & \rho(x) \geqslant \delta, \\
\delta, & \text { если } & \rho(x)<\delta,
\end{array}\right. \\
\rho_{* j}:=\left.\bar{\rho}_{*}\right|_{\Gamma_{j}}=\frac{1}{\gamma_{j}} \int_{\Gamma_{j}} \rho_{0} d \mu, \quad s_{*}=\mathbf{S}\left(\rho_{*}\right), \quad \bar{s}_{*}=\mathbf{S}\left(\bar{\rho}_{*}\right) .
\end{gathered}
$$

Имеем

$$
0 \leqslant s-s_{*} \leqslant \delta \ln \delta
$$

Если диаметр разбиения $\left\{\Gamma_{j}\right\}$ достаточно мал, то согласно лемме 2 выполнены неравенства

$$
0<\bar{s}_{*}-s_{*} \leqslant \delta
$$

Таким образом, достаточно доказать, что

$$
\bar{s}<\bar{s}_{*}+c \delta,
$$


где $c>0$ - постоянная, не зависящая от $\delta$. Действительно, тогда ввиду (П.9), (П.10) будем иметь $\left|s-\bar{s}_{*}\right|<\delta+\delta \ln \delta$. Следовательно, из (П.2) и (П.11) вытекает, что $s \leqslant \bar{s} \leqslant \bar{s}_{*}+c \delta \leqslant s+(1+c) \delta+\delta \ln \delta$, откуда получаем $|s-\bar{s}|<(1+c) \delta+\delta \ln \delta$.

Проверим справедливость оценки (П.11). Согласно определению функции $\rho_{*}$ имеem

$$
\delta \leqslant \rho_{*} \leqslant \rho+\delta \leqslant \Delta+\delta .
$$

Следовательно, $\delta \leqslant \bar{\rho}_{*} \leqslant \bar{\rho}+\delta \leqslant \Delta+\delta$. Из неравенства

$$
\sup _{\rho \in(0, \Delta+\delta)}(\rho \ln \rho)^{\prime}=1+\ln (\Delta+\delta)
$$

вытекает, что $\bar{\rho}_{*} \ln \bar{\rho}_{*} \leqslant \bar{\rho} \ln \bar{\rho}+(1+\ln (\Delta+\delta)) \delta$, откуда получаем

$$
\bar{s} \leqslant \bar{s}_{*}+(1+\ln (\Delta+1)) \delta .
$$

П.4. Доказательство теоремы 2: часть 3. В этом пункте мы доказываем лемму 2. Зафиксируем произвольное $\varepsilon>0$. Так как $C^{0}(\Gamma)$ плотно в $L_{1}(\Gamma, \mu)$, найдется функция $\rho_{c} \in C^{0}(\Gamma)$ такая, что

$$
\delta<\rho_{c}<\Delta, \quad \rho=\rho_{c}\left(1+\rho_{l}\right), \quad\left\|\rho_{l}\right\|<\varepsilon
$$

Здесь $\|\cdot\|-L_{1}$-норма. Тогда

$$
\begin{gathered}
s=-\int_{\Gamma} \rho_{c}\left(1+\rho_{l}\right) \ln \left(\rho_{c}\left(1+\rho_{l}\right)\right) d \mu=s_{c}+A_{1}+A_{2}, \\
s_{c}:=\mathbf{S}\left(\rho_{c}\right), \quad A_{1}=-\int_{\Gamma} \rho \ln \left(1+\rho_{l}\right) d \mu, \quad A_{2}=-\int_{\Gamma} \rho_{c} \rho_{l} \ln \rho_{c} d \mu .
\end{gathered}
$$

Таким образом, остается проверить, что величины $\left|\bar{s}-s_{c}\right|, A_{1}$ и $A_{2}$ малы.

Так как $\left|\rho_{c} \ln \rho_{c}\right| \leqslant \Delta \ln \Delta$, имеем

$$
\left|A_{2}\right| \leqslant \Delta \ln \Delta \int_{\Gamma}\left|\rho_{l}\right| d \mu<\varepsilon \Delta \ln \Delta .
$$

Согласно (П.12) $\rho_{l}=\left(\rho-\rho_{c}\right) / \rho_{c} \in I$, где $I=[-1+\delta / \Delta, 1+\Delta / \delta]$. Несложно установить, что

$$
\max _{\rho \in I}\left|\frac{\ln (1+\rho)}{\rho}\right| \leqslant \ln \frac{\Delta}{\delta} .
$$

Следовательно,

$$
\left|A_{1}\right| \leqslant \Delta \int_{\Gamma}\left|\ln \left(1+\rho_{l}\right)\right| d \mu \leqslant \Delta \ln \frac{\Delta}{\delta} \int_{\Gamma}\left|\rho_{l}\right| d \mu \leqslant \varepsilon \Delta \ln \frac{\Delta}{\delta} .
$$

Функция $\rho_{c}$ непрерывна на компакте $\Gamma$, следовательно, она равномерно непрерывна, т.е. существует $\sigma>0$ такое, что $\left|\rho_{c}\left(z_{1}\right)-\rho_{c}\left(z_{2}\right)\right|<\varepsilon$ для любых $z_{1}, z_{2} \in \Gamma$ таких, что $\operatorname{dist}\left(z_{1}, z_{2}\right)<\sigma$. 
Пусть $\operatorname{diam} \Gamma_{j}<\sigma$. Тогда $\left|\bar{\rho}_{c}-\rho_{c}\right|<\varepsilon$. Имеем оценку

$$
\begin{aligned}
\left|\bar{s}_{c}-s_{c}\right| & =\left|\int_{\Gamma}\left(\rho_{c} \ln \rho_{c}-\bar{\rho}_{c} \ln \bar{\rho}_{c}\right) d \mu\right| \leqslant \\
& \leqslant\left(1+\ln \frac{\Delta}{\delta}\right) \int_{\Gamma}\left|\rho_{c}-\bar{\rho}_{c}\right| d \mu \leqslant\left(1+\ln \frac{\Delta}{\delta}\right) \varepsilon .
\end{aligned}
$$

Положим $\rho_{c j}=\left.\bar{\rho}_{c}\right|_{\Gamma_{j}}$. Тогда

$$
\begin{gathered}
\left|\rho_{j}-\rho_{c j}\right|=\left|\frac{1}{\gamma_{j}} \int_{\Gamma_{j}} \rho_{c} \rho_{l} d \mu\right| \leqslant \frac{\Delta}{\gamma_{j}} r_{j}, \\
r_{j}=\int_{\Gamma_{j}}\left|\rho_{l}\right| d \mu, \quad \sum_{j \in J} r_{j}<\varepsilon .
\end{gathered}
$$

Следовательно, для любого $j \in J$

$$
\left|\rho_{j} \ln \rho_{j}-\rho_{c j} \ln \rho_{c j}\right| \leqslant\left(1+\ln \frac{\Delta}{\delta}\right)\left|\rho_{j}-\rho_{c j}\right| \leqslant \frac{\Delta}{\gamma_{j}}\left(1+\ln \frac{\Delta}{\delta}\right) r_{j} .
$$

Получаем следующую оценку для разности энтропий:

$$
\left|\bar{s}_{c}-\bar{s}\right|=\left|\sum_{j \in J}\left(\rho_{j} \ln \rho_{j}-\rho_{c j} \ln \rho_{c j}\right) \gamma_{j}\right| \leqslant \Delta\left(1+\ln \frac{\Delta}{\delta}\right) \varepsilon .
$$

Из (П.15), (П.16) находим

$$
\left|\bar{s}-s_{c}\right| \leqslant(1+\Delta)\left(1+\ln \frac{\Delta}{\delta}\right) \varepsilon .
$$

П.5. Доказательство теоремы 3. Очевидно, можно считать, что $d<1$. Пусть $\varepsilon>0$ произвольно. Положим

$$
\hat{J}_{l}=\left\{j \in J: \Gamma_{j} \subset K_{l}\right\}, \quad \widehat{K}_{l}=\bigcup_{j \in \hat{J}_{l}} \Gamma_{j} .
$$

Тогда для достаточно больших $N$ выполняются неравенства

$$
\begin{aligned}
& \left|s-\int_{\widehat{K}_{N}} \rho \ln \rho d \mu\right|<\varepsilon, \\
& \left|\bar{s}-\int_{\widehat{K}_{N}} \bar{\rho} \ln \bar{\rho} d \mu\right|<\varepsilon .
\end{aligned}
$$

Действительно, проверим (П.17):

$$
\begin{gathered}
\left|s-\int_{\widehat{K}_{N}} \rho \ln \rho d \mu\right| \leqslant A_{N}+A_{N+1}+\cdots, \\
A_{l}=\int_{\widehat{K}_{l+1} \backslash \widehat{K}_{l}}|\rho \ln \rho| d \mu .
\end{gathered}
$$


Для любого $l>1$ выполнены включения $K_{l-1} \subset \widehat{K}_{l} \subset K_{l}$ (второе включение следует из определения множества $\widehat{K}_{l}$, а первое - из определения $1($ г) и неравенства $d<1$ ). Таким образом,

$$
\widehat{K}_{l+1} \backslash \widehat{K}_{l} \subset K_{l+1} \backslash K_{l-1} .
$$

Следовательно, согласно определению 1 (в)

$$
\mu\left(\widehat{K}_{l+1} \backslash \widehat{K}_{l}\right) \leqslant \mu\left(K_{l+1} \backslash K_{l}\right)+\mu\left(K_{l} \backslash K_{l-1}\right) \leqslant 2 C l^{n-1} .
$$

Из условия (3) теоремы вытекает, что

$$
\left.\rho\right|_{\widehat{K}_{l+1} \backslash \widehat{K}_{l}} \leqslant\left.\rho\right|_{K_{l+1}}<c_{\rho}(l+1)^{-n-\delta} .
$$

Так как можно считать, что $c_{\rho}(N+1)^{-n-\delta}<1 / e$, имеем

$$
A_{l} \leqslant 2 C l^{n-1} c_{\rho}(l+1)^{-n-\delta}\left|\ln \left(c_{\rho}(l+1)^{-n-\delta}\right)\right|
$$

для всех $l \geqslant N$. Неравенство (П.17) при достаточно больших $N$ вытекает из оценки (П.19). Неравенство (П.18) доказывается аналогично.

Согласно определению 1 (в) $\mu\left(\widehat{K}_{N}\right)<\infty$. Поэтому из теоремы 1 следует, что при достаточно малых $d>0$

$$
\left|\int_{\widehat{K}_{N}} \rho \ln \rho d \mu-\int_{\widehat{K}_{N}} \bar{\rho} \ln \bar{\rho} d \mu\right|<\varepsilon .
$$

Из неравенств (П.17), (П.18) и (П.20) вытекает, что при достаточно малых $d>0$ $|s-\bar{s}|<3 \varepsilon$, что и требовалось доказать.

П.6. Доказательство теоремы 7. Отметим, что в доказательстве нигде не используется то, что $\rho \geqslant 0$. Поэтому, заменяя $\rho$ на $\rho-\langle\rho\rangle$, видим, что можно ограничиться рассмотрением случая $\langle\rho\rangle=0$.

Рассмотрим при больших $t$ функции

$$
\rho_{k}(t, x)=M^{n} \int_{\Gamma_{k}^{\omega}} \rho(x+\omega t, \omega) d \omega=\frac{M^{n}}{t^{n}} \int_{t \Gamma_{k}^{\omega}} \rho\left(x+\beta, \frac{\beta}{t}\right) d \beta,
$$

где

$$
t \Gamma_{k}^{\omega}=\left\{\beta \in \mathbb{R}^{n}: \frac{t k_{l}}{M} \leqslant \beta_{l} \leqslant \frac{t\left(k_{l}+1\right)}{M}, l=1, \ldots, n\right\} .
$$

ЛЕмма П.1. Если $\langle\rho\rangle=0$, mo

$$
\left|\rho_{k}(t, x)\right| \leqslant \frac{n M}{t}\left(\frac{\|\rho\|_{1}}{M}+2\|\rho\|_{0}\right) .
$$

Теорема 7 сразу следует из леммы П.1, так как при $\langle\rho\rangle=0$

$$
\left|\rho_{j k}(t)\right|=\left|\int_{\mathbb{T}^{n}} \rho_{k}(t, x) d x\right| .
$$


ДоКАЗАТЕЛЬСТво ЛЕммы П.1. Представим множество $t \Gamma_{k}^{\omega}$ в виде объединения единичных кубов $\mathcal{C}_{m}, m \in \mathcal{Z}$, и остатка $R$. Здесь $\mathcal{Z}=\mathcal{Z}(t)$ - конечное подмножество $\mathbb{Z}^{n}$,

$$
\mathcal{C}_{m}=\left\{\beta \in \mathbb{R}^{n}: m_{l} \leqslant \beta_{l} \leqslant m_{l}+1, l=1, \ldots, n\right\}
$$

и $R=t \Gamma_{k}^{\omega} \backslash \bigcup_{m \in \mathcal{Z}} \mathcal{C}_{m}$. Считаем, что $R$ не содержит целиком ни одного куба $\mathcal{C}_{m}$. Тогда

$$
\int_{R} d \beta \leqslant \frac{2 n t^{n-1}}{M^{n-1}}, \quad \# \mathcal{Z} \leqslant \frac{t^{n}}{M^{n}}
$$

Имеем

$$
\begin{gathered}
\rho_{k}=\frac{1}{M^{n} t^{n}}\left(\sum_{m \in \mathcal{Z}} I_{m}+I_{R}\right), \\
I_{m}=\int_{\mathcal{C}_{m}} \rho\left(x+\beta, \frac{\beta}{t}\right) d \beta, \quad I_{R}=\int_{R} \rho\left(x+\beta, \frac{\beta}{t}\right) d \beta .
\end{gathered}
$$

Так как $\langle\rho\rangle=0$, то для любого $\beta_{0} \in \mathbb{R}^{n}$ выполнено равенство

$$
\int_{\mathcal{C}_{m}} \rho\left(x+\beta, \frac{\beta}{t}\right) d \beta=0 .
$$

Поэтому

$$
\left|I_{m}\right|=\left|\int_{\mathcal{C}_{m}}\left(\rho\left(x+\beta, \frac{\beta}{t}\right)-\rho\left(x+\beta, \frac{m}{t}\right)\right) d \beta\right| \leqslant \int_{\mathcal{C}_{m}} \frac{n\|\rho\|_{1}}{t} d \beta=\frac{n\|\rho\|_{1}}{t} .
$$

С другой стороны, $|R| \leqslant \int_{R}\|\rho\|_{0} d \beta$. Следовательно, используя (П.21) и (П.22), получаем

$$
\left|\rho_{k}(t, x)\right| \leqslant \frac{M^{n}}{t^{n}}\left(n\|\rho\|_{1} \frac{t^{n-1}}{M^{n}}+2 n\|\rho\|_{0} \frac{t^{n-1}}{M^{n-1}}\right)=\frac{n M}{t}\left(\frac{\|\rho\|_{1}}{M}+2\|\rho\|_{0}\right) .
$$

Лемма доказана.

Благодарности. Авторы благодарны В. В. Сидоренко и О. Г. Смолянову за полезные обсуждения. Работа выполнена при финансовой поддержке РФФИ (гранты № 05-01-01058, № 05-01-01119), Программы поддержки ведущих научных школ (грант № НШ-1312.2006.1) и программы президиума РАН "Нелинейная динамика".

\section{Список литературы}

[1] Р. Боуэн, Методы символической динамики, Мир, М., 1979.

[2] М. Кац, Вероятность и смежсные вопросы в физике, Мир, М., 1965.

[3] Н. С. Крылов, Работы по обоснованию статистической физики, Изд-во АН СССР, M.-Л., 1950.

[4] А. Пуанкаре, “Замечания о кинетической теории газов”, Избранные труды, т. III, Наука, М., 1974.

[5] Дж. В. Гиббс, Термодинамика. Статистическая механика, Наука, М., 1982.

[6] В. В. Козлов, Д. В. Трещев, ТМФ, 136:3 (2003), 496-506.

[7] В. В. Козлов, Д. В. Трещев, ТМФ, 134:3 (2003), 388-400.

[8] С. В. Гонченко, Д. В. Тураев, Л. П. Шильников, Докл. РАН, 407:3 (2006), 299-303. 